\title{
Crisis management during anaesthesia: water intoxication
}

\author{
M T Kluger, S M Szekely, R J Singleton, S C Helps
}

Qual Saf Health Care 2005;14:e23 (http://www.qshc.com/cgi/content/full/14/3/e23). doi: 10.1136/qshc.2002.004242

Background: Irrigation of closed body spaces may lead to substantial perioperative fluid and electrolyte shifts. A syndrome occurring during transurethral resection of prostate (TURP), and a similar syndrome described in women undergoing transcervical endometrial ablation (TCEA) are both characterised by a spectrum of symptoms which may range from asymptomatic hyponatraemia to convulsions, coma, and death. Such potentially serious consequences require prompt recognition and appropriate management of this "water intoxication" syndrome.

Objectives: To examine the role of a previously described core algorithm "COVER ABCD-A SWIFT CHECK", supplemented by a specific sub-algorithm for water intoxication, in the management of this syndrome occurring in association with anaesthesia.

Methods: The potential performance of this structured approach for each of the relevant incidents among

See end of article for authors' affiliations

.....................

Correspondence to: Professor W B Runciman, President, Australian Patient Safety Foundation GPO Box 400, Adelaide, South Australia, 5001, Australia; research@apsf. net.au

Accepted 12 January 2005 the first 4000 reported to the Australian Incident Monitoring Study (AIMS) was compared with the actual management as reported by the anaesthetists involved.

Results: From the first 4000 incidents reported to AIMS, 10 reports of water intoxication were identified, two from endometrial ablations under general anaesthesia and eight from male urological procedures under spinal anaesthesia. The "core" crisis management algorithm detected a problem in seven cases; however, it was deficient in dealing with neurological presentations. Diagnosis of the cause of the incident would have required a specific water intoxication sub-algorithm in eight cases and a hypotension algorithm in a further two cases. Corrective strategies also required a specific sub-algorithm in eight cases, while the hypotension and cardiac arrest sub-algorithms were required in conjunction with the water intoxication sub-algorithm in the remaining two.

Conclusion: This relatively uncommon problem is managed poorly using the "core" crisis management sub-algorithm and requires a simple specific sub-algorithm for water intoxication. p rigation of closed body spaces may lead to substantial perioperative fluid and electrolyte shifts. The transurethral resection of prostate (TURP) syndrome is characterised by a spectrum of symptoms ranging from asymptomatic hyponatraemia, to electrocardiographic (ECG) changes, nausea, vomiting, convulsions, coma, and death. ${ }^{12}$ A similar syndrome has been described in women undergoing transcervical endometrial ablation (TCEA). ${ }^{3}$ The occurrence of this "water intoxication" syndrome is determined by a combination of operator, patient, and procedural factors. Asymptomatic hyponatraemia can occur in over 50\% of TURPs, while clinically detectable TURP syndrome may become obvious in $2 \%$ of resections. ${ }^{5}$ As the consequences of this syndrome can be serious, prompt recognition and appropriate management are important.

The aim of this study was to identify all cases of water intoxication in the first 4000 incidents reported to the Australian Incident Monitoring Study (AIMS), create a management algorithm, and compare this with the actual management of the incidents described.

In 1993, a "core" crisis management algorithm, represented by the mnemonic COVER ABCD-A SWIFT CHECK (the $\mathrm{AB}$ precedes COVER for the non-intubated patient) was proposed as the basis for a systematic approach to any crisis during anaesthesia where it is not immediately obvious what should be done, or where actions taken have failed to remedy the situation. ${ }^{6}$ This was validated against the first 2000 incidents reported to the original AIMS. AIMS is an ongoing study which involves the voluntary, anonymous reporting of any unintended incident which reduced, or could have reduced the safety margin for a patient. ${ }^{7}$

It was concluded that if this algorithm had been correctly applied, a functional diagnosis would have been reached within 40-60 seconds in 99\% of applicable incidents, and that the learned sequence of actions recommended by the COVER portion would have led to appropriate steps being taken to handle the $60 \%$ of problems relevant to this portion of the algorithm. ${ }^{7}$ However, this study also showed that the $40 \%$ of problems represented by the remainder of the algorithm, ABCD-A SWIFT CHECK, were not always promptly diagnosed or appropriately managed..$^{6-8}$ It was decided that it would be useful for these remaining problems to develop a set of sub-algorithms in an easy to use crisis management manual. ${ }^{9}$ This study reports on the place of the COVER ABCD-A SWIFT CHECK algorithm in the diagnosis and initial management of water intoxication, provides an outline of a specific crisis management sub-algorithm for this problem during anaesthesia, and provides an indication of the potential value of using this structured approach.

\section{METHODS}

Of the first 4000 incidents reported to AIMS, those which made reference to water intoxication, glycine, irrigation, urology, endometrial surgery, hyponatraemia, low sodium, and TURP were extracted and analysed for relevance, presenting features, type of surgery, cause, management, and outcome.

The COVER ABCD-A SWIFT CHECK algorithm, described elsewhere in this set of articles ${ }^{9}$ was applied to each relevant report to determine the stages at which the problem might have been diagnosed and to confirm that activating the COVER portion would have led to appropriate initial steps being taken. As water intoxication is not adequately dealt with by this algorithm, a specific sub-algorithm for this syndrome was developed (see figure) and its putative effectiveness was tested against the reports. How this was 
done is described elsewhere in this set of articles. ${ }^{9}$ The potential value of this structured approach (that is, the application of COVER ABCD-A SWIFT CHECK) to the diagnosis and initial management of the problem, followed by the application of the water intoxication sub-algorithm, was assessed in the light of AIMS reports by comparing its potential effectiveness for each incident with that of the actual management, as recorded in each report.

\section{RESULTS}

Ten incidents of water intoxication were identified. The majority had undergone transurethral procedures on either prostate (7) or bladder (1), all under spinal blockade. Two incidents involved TCEA, both of which were performed under general anaesthesia. The presenting features are shown in table 1.

When the COVER ABCD-A SWIFT CHECK algorithm was applied to each report, it was considered that the majority of cases $(70 \%)$ would have been detected at the COVER portion of the algorithm. The actions recommended by the COVER portion (100\% oxygen, turning off the vaporiser, and, if necessary, removing the patient from the anaesthetic machine, filter, and circuit) were all considered reasonable immediate steps. However diagnosis of the actual problem
Table 1 Presenting features of water intoxication/ hyponatraemia*

\begin{tabular}{|c|c|c|}
\hline & $\begin{array}{l}\text { General anaesthesia } \\
\text { (GA) } \dagger\end{array}$ & $\begin{array}{l}\text { Regional anaesthesia } \\
\text { (RA)t† }\end{array}$ \\
\hline $\begin{array}{l}\text { Confusion, seizure, } \\
\text { sedation/drowsiness }\end{array}$ & $N / A$ & 6 \\
\hline Chest pain, ECG changes & 2 & 4 \\
\hline Hypotension & 0 & 2 \\
\hline Bronchospasm & 1 & 1 \\
\hline Desaturation & 2 & 0 \\
\hline
\end{tabular}

*Some patients presented with more than one symptom or sign. †All endometrial ablations $(n=7)$ were performed under $G A$, and all TURP/bladder procedures $(n=8)$ were performed under RA. $\ddagger$ There was one case each of hypertension and bradycardia in this subgroup.

was only possible from COVER in two cases while the other eight required specific consideration. Effective treatment required a specific sub-algorithm (fig 1) in eight cases; one case required both hypotension ${ }^{10}$ and water intoxication subalgorithms while the final case would have required the use of sub-algorithms for cardiac arrest, ${ }^{11}$ hypotension, ${ }^{10}$ and water intoxication.

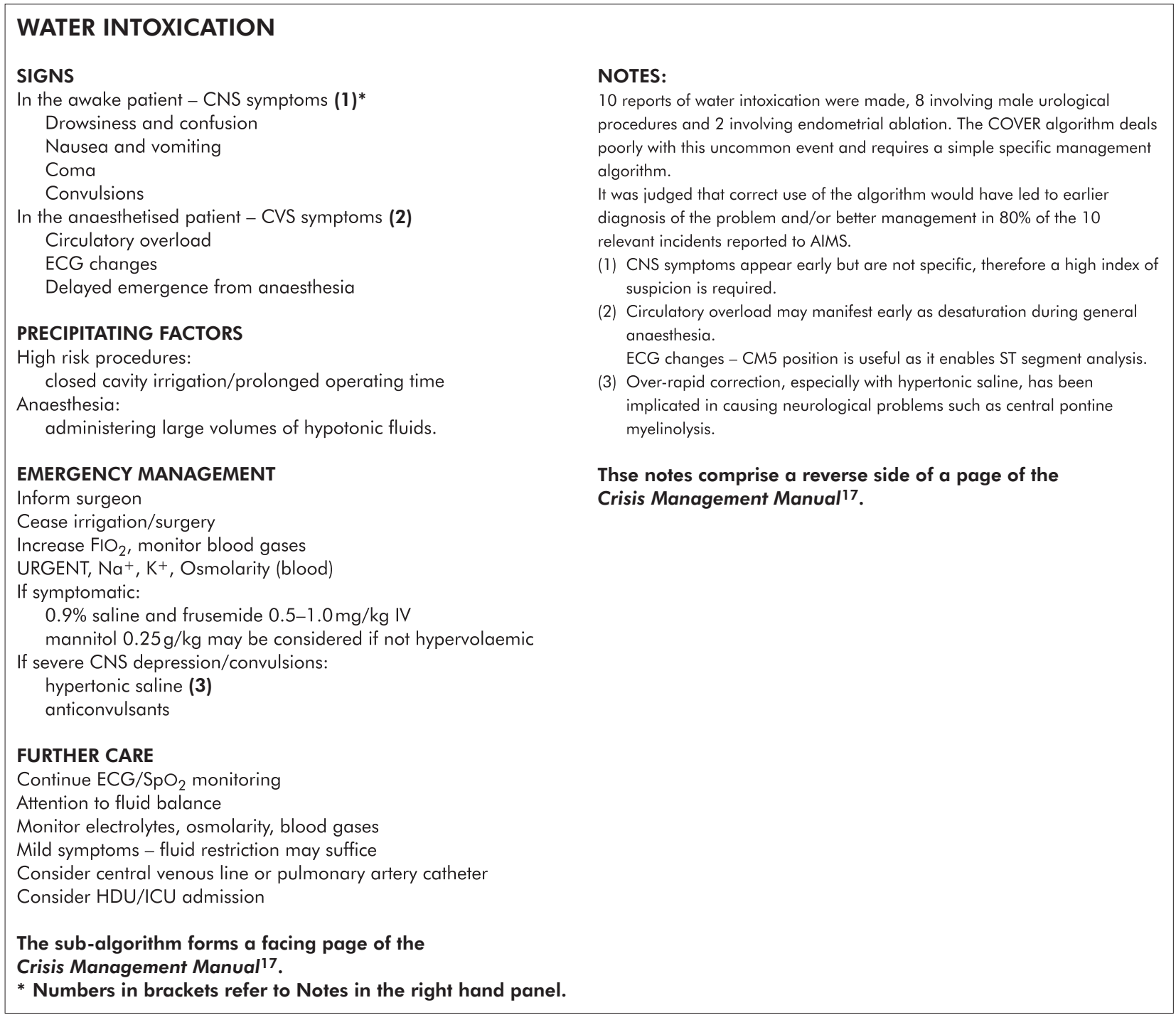

Figure 1 Water intoxication. 
The sub-algorithm (shown in fig l, left hand panel) was estimated to be as effective as the actual management in seven cases; in the remaining three cases, earlier consideration and action may have improved outcome. In one incident there was a delay in diagnosis and the operation continued despite symptoms of confusion, nausea, vomiting, and chest pain. In another case pre-existing hyponatraemia ( $129 \mathrm{mmol} / \mathrm{l}$ ) was left uncorrected, and this considerably worsened following a lengthy cystoscopy $(98 \mathrm{mmol} / \mathrm{l})$ and finally a protracted (three hour) resection led to increasing drowsiness and hypertension. The attending anaesthetist did not realise the significance of these signs until a relief consultant took over the case.

There was minor morbidity in three cases, with major morbidity in seven. There were no deaths. Half of all the patients with water intoxication were admitted to intensive care or high dependency units. The management of these cases is summarised in table 2. Presenting blood pressure was variable; hypotension in thee cases, hypertension in one; normotension in three, and unstated in three.

\section{DISCUSSION}

The small number of reports analysed for this survey limits the conclusions that can be drawn. A significant proportion of presenting features of water intoxication appear to be neurological signs; these were poorly managed using the COVER ABCD algorithm and hence a specific sub-algorithm needed to be developed. The majority of cases of water intoxication present with neurological signs in awake patients or with cardiovascular signs in patients under general anaesthesia. Although the term "water intoxication" is used, it is appreciated that the signs and symptoms may be related to fluid overload, hyponatraemia, glycine, or ammonia toxicity. ${ }^{1}$ It was judged that correct use of the subalgorithm for water intoxication, once the possibility was considered, would have confirmed the diagnosis in all cases, by checking the serum sodium and osmolality, and would have led to definitive management in eight of 10 reports. The remaining two incidents also required a sub-algorithm for hypotension. Some general conclusions may be drawn from this small study.

\section{Precipitating factors}

The surgical procedures that predispose patients to water intoxication are chiefly transurethral resection of the prostate and endometrial ablation.

\section{Clinical features}

Under spinal anaesthesia, neurological signs appear early and include nausea, vomiting, confusion, and irritability. A high index of suspicion is needed when a patient's neurological condition changes intraoperatively during one of these procedures. Further sedation should be avoided and the diagnosis of metabolic derangement considered; serum sodium and osmolarity should be checked.

The earliest signs under general anaesthesia appear to be cardiorespiratory, including desaturation and ECG changes. These are well recognised as indicative of potential intraoperative problems in TURP. ${ }^{12}$ Full ECG monitoring therefore is advocated with lead positioning (for example, CM5) that enables ST segment analysis. In addition, oximetry should be mandatory for all patients.

\section{Management}

Avoidance of excessive resection time, irrigation fluid pressure, and administration of hypotonic fluids may prevent many cases of water intoxication. Immediate cessation of the procedure is warranted in all cases where this problem arises. Continuation may lead to a worsening of the clinical situation and potential complications. If the symptoms are mild, blood should be taken for urgent electrolyte and osmolality measurement. Often no further active treatment is required. If patients have neurological signs or exhibit signs of fluid overload, consideration needs to be given to the use of a diuretic (for example, frusemide), and fluid restriction. Mannitol has been found to be more effective than frusemide in the treatment of TURP syndrome as reflected in higher serum sodium and less need for postoperative fluid volume loading. ${ }^{13}$ However, in patients who are already fluid overloaded, mannitol may precipitate an initial worsening of this situation; in these, frusemide should be used. ${ }^{14}$ Normalisation of oxygenation and cardiovascular parameters should be sought. As the TURP patients are in an older age group, acute changes in intravascular fluid shifts may warrant invasive arterial and central venous or pulmonary artery pressure monitoring. Avoidance of hypotonic solutions and administration of isotonic saline is recommended. Hypertonic saline may occasionally be warranted, especially when the serum sodium falls below $120 \mathrm{mmol} / \mathrm{l}$. Its use is required only in those cases where there is severe, acute neurological deterioration (for example, convulsions and coma); however overly rapid correction, especially with prexisiting hyponatraemia, has been implicated in causing neurological problems such as central pontine myelinolysis. ${ }^{15}$ As half of these patients required ICU or HDU admission, consideration should be given to admission of these patients to the appropriate ward following the procedure.

The mainstays of treatment of water intoxication include awareness of the problem in high risk patient groups, intraoperative electrolyte and blood gas monitoring and early cessation of surgery if the complication is considered. Marker techniques such as ethanol added to irrigation fluid have

Table 2 Management of water intoxication/hyponatraemia/glycine intoxication

\begin{tabular}{llllc}
\hline Case & Anaesthetic & Case & Management & Lowest Na (mmol/I) \\
\hline 1 & GA & TCEA & CVP line, frusemide & 99 \\
2 & SP & TURP & Frusemide; normal saline & 121 \\
3 & SP & TURP & Frusemide; normal saline & 115 \\
4 & SP & TURP & CPR; adrenaline; atropine & 112 \\
5 & SP & TURP & Hypertonic saline; hydrocortisone & 117 \\
6 & SP & BNI & Frusemide; GTN patch & 98 \\
7 & SP & TURP & Frusemide; hypertonic saline; CVP line & 115 \\
8 & SP & TURP & IPPV (convulsion) & 130 \\
9 & SP & TURP & IPPV (pulmonary oedema); frusemide & "low" \\
10 & GA & TCEA & Frusemide & 123 \\
\hline
\end{tabular}

GA, general anaesthesia; SP, spinal anaesthesia; TCEA, endometrial ablation; TURP, transurethral resection of prostate; BNI, bladder neck incision; CVP, central venous pressure; CPR, cardiopulmonary resuscitation; GTN, glyceryl trinitrate; IPPV, intermittent positive pressure ventilation. 


\section{Key messages}

- There were 10 reports of water intoxication identified from the first 4000 reports to the Australian Incident Monitoring Study (AIMS).

- Eight cases were associated with transurethral procedures under spinal anaesthesia. Two cases were associated with transcervical endometrial ablation (TCEA).

- The lowest recorded serum sodium level was $99 \mathrm{mmol} / \mathrm{l}$, during a TCEA.

- There were no deaths but there was major morbidity in seven cases.

- The commonest presenting signs in the awake patient were developing mental confusion, sedation, drowsiness, and seizure. The earliest signs under general anaesthesia appear to be cardiorespiratory (for example, desaturation, ECG changes). Presenting blood pressure was variable. Electrocardiographic and pulse oximetry monitoring are considered mandatory.

- The commonest management strategies reported involved administration of frusemide, normal saline, and IPPV.

- Half the patients required ICU or HDU admission postoperatively, indicating the need for admission to the appropriate ward following these procedures.

- Risk situations during transurethral or TCEA procedures include prolonged surgical time and excessive pressures using hypotonic irrigation fluids.

- The combined, correct application of the core algorithm and the specific sub-algorithm was considered likely to have improved the outcome for three of the patients.

proven effective in the early detection of this problem in TURP patients. ${ }^{514}$ Newer techniques of monitoring intravesical bladder pressure along with developments in resection techniques (for example, laser, minimally invasive surgery) may also help to reduce the incidence of this problem. ${ }^{16}$ The role of aggressive monitoring and hypertonic saline, however, requires further study. Although COVER is able to detect the presence of a problem, diagnosis and management of water intoxication requires a high index of suspicion and the use of a specific sub-algorithm.

Finally, it is important that a full explanation of what happened be given to the patient and the problem clearly documented in the anaesthetic record. If a particular precipitating event was significant, or a particular action was useful in resolving the crisis, this should be clearly explained and documented.

\section{ACKNOWLEDGEMENTS}

The authors would like to thank all the anaesthetists in Australia and New Zealand who contributed to the 4000 incident reports upon which this and the other 24 papers in the Crisis Management Series are based. The coordinators of the project also thank Liz Brown for preparing the draft of the original Crisis Management Manual; Loretta Smyth for typing; Monika Bullock, RN, for earlier coding and classifying of data; Dr Charles Bradfield for the electronic version of the algorithms; Dr Klee Benveniste for literature research; Drs Klee Benveniste, Michal Kluger, John Williamson, and Andrew Paix for editing and checking manuscripts.

\section{Authors' affiliations}

M T Kluger, Senior Staff Specialist, Department of Anaesthesiology and Perioperative Medicine, North Shore Hospital, Auckland, New Zealand S M Szekely, Senior Staff Specialist, Department of Anaesthesia and Intensive Care, Royal Adelaide Hospital and University of Adelaide, Adelaide, South Australia, Australia

R J Singleton, Senior Staff Specialist, Department of Anaesthesia and Intensive Care, Royal Adelaide Hospital and University of Adelaide, Adelaide, South Australia, Australia

S C Helps, Metabolic Neurochemistry Unit, Department of Medical Biochemistry, School of Medicine, Flinders University, Bedford Park, South Australia, Australia

This study was coordinated by the Australian Patient Safety Foundation, GPO Box 400, Adelaide, South Australia, 5001, Australia.

\section{REFERENCES}

1 Jensen V. The TURP syndrome. Can J Anaesth 1991;38:90-6.

2 Hughes PD, McNicol D, Mutton PM, et al. Postoperative hyponatraemic encephalopathy: water intoxication. Aust NZ J Surg 1998;68:165-8.

3 McSwiney M, Myatt J, Hargreaves M. Transcervical endometrial resection syndrome. Anaesthesia 1995;50:254-7.

4 Hwang CB, Hui YL, Liu WC, et al. Absorption of irrigating fluid during transcervical resection of endometrium - a report of two cases. Acta Anaesthesiol Sin 1997:35:45-50.

5 Okeke AA, Lodge R, Hinchliffe A, et al. Ethanol-glycine irrigating fluid for transurethral resection of the prostate in practice. BJU Int 2000;86:43-6.

6 Runciman WB, Webb RK, Klepper ID, et al. Crisis management: validation of an algorithm by analysis of 2000 incident reports. Anaesth Intensive Care 1993:21:579-92.

7 Webb RK, Currie M, Morgan CA, et al. The Australian Incident Monitoring Study: an analysis of 2000 incident reports. Anaesth Intensive Care 1993;21:520-8.

8 Webb RK, van der Walt JH, Runciman WB, et al. Which monitor? An analysis of 2000 incident reports. Anaesth Intensive Care 1993;21:529-42.

9 Runciman WB, Kluger MT, Morris RW, et al. Crisis management during anaesthesia: the development of an anaesthetic crisis management manual. Qual Saf Health Care 2005; 14:e1

10 Morris RW, Watterson LM, Westhorpe RN, et al. Crisis management during anaesthesia: hypotension. Qual Saf Health Care 2005;14:e11.

11 Runciman WB, Morris RW, Watterson LM, et al. Crisis management during anaesthesia: cardiac arrest. Qual Saf Health Care 2005; 14:e14.

12 Hahn RG, Essen P. ECG and cardiac enzymes after glycine absorption in transurethral prostatic resection. Acta Anaesthesiol Scand 1994;38:550-6.

13 Crowley K, Clarkson K, Hannon V, et al. Diuretics after transurethral prostatectomy: a double blind controlled trial comparing frusemide and mannitol. Br J Anaesth 1990;65:337-41.

14 Hahn RG. Early detection of the TUR syndrome by marking the irrigating fluid with 1\% ethanol. Acta Anaesthesiol Scand 1989:33:146-51

15 Sterns RH, Riggs JE, Schochet SS. Osmotic demyelination syndrome following correction of hyponatraemia. N Engl J Med 1986;314:1535-42.

16 Gray RA, Lynch C, Hehir M, Worsley M. Intravesical pressure and the TUR syndrome. Anaesthesia, 2001;56:461-5.

17 Australian Patient Safety Foundation. Crisis Management Manual: COVER $A B C D$ A SWIFT CHECK. Adelaide: Australian Patient Safety Foundation, 1996, 74pp. Available at http://www.apsf.net.au/anaesthesia.htm (accessed 6 September 2004). 